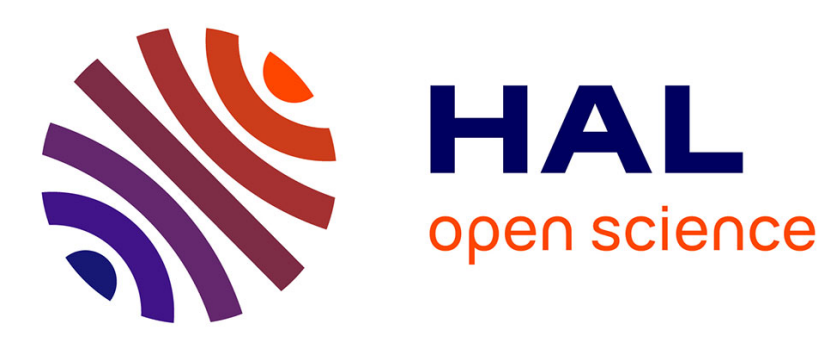

\title{
A Curvature Tensor Distance for Mesh Visual Quality Assessment
}

\author{
Fakhri Torkhani, Kai Wang, Jean-Marc Chassery
}

\section{To cite this version:}

Fakhri Torkhani, Kai Wang, Jean-Marc Chassery. A Curvature Tensor Distance for Mesh Visual Quality Assessment. ICCVG 2012 - International Conference on Computer Vision and Graphics, Sep 2012, Warsaw, Poland. 253-263, 11 p. hal-00719315

\section{HAL Id: hal-00719315 https://hal.science/hal-00719315}

Submitted on 19 Jul 2012

HAL is a multi-disciplinary open access archive for the deposit and dissemination of scientific research documents, whether they are published or not. The documents may come from teaching and research institutions in France or abroad, or from public or private research centers.
L'archive ouverte pluridisciplinaire HAL, est destinée au dépôt et à la diffusion de documents scientifiques de niveau recherche, publiés ou non, émanant des établissements d'enseignement et de recherche français ou étrangers, des laboratoires publics ou privés. 


\title{
A Curvature Tensor Distance for Mesh Visual Quality Assessment
}

\author{
Fakhri Torkhani, Kai Wang, and Jean-Marc Chassery \\ Gipsa-lab, CNRS UMR 5216, Grenoble, France \\ firstname. lastname@gipsa-lab.grenoble-inp.fr
}

\begin{abstract}
This paper presents a new objective metric for assessing the visual difference between a reference or 'perfect' mesh and its distorted version. The proposed metric is based on the measurement of a distance between curvature tensors of the two triangle meshes under comparison. Unlike existing methods, our algorithm uses not only eigenvalues but also eigenvectors of the curvature tensor to derive a perceptually-oriented distance. Our metric also accounts for some important properties of the human visual system. Experimental results show good coherence between the proposed objective metric and subjective assessments.
\end{abstract}

\section{Introduction}

Three-dimensional (3D) meshes are now used in many multimedia applications such as digital entertainment, medical imaging and computer-aided design. It is common that 3D meshes undergo some lossy operations like simplification, compression and watermarking. Since the end users are often human beings, it is thus important to derive metrics that can faithfully evaluate the perceptual distortions introduced by such operations [1]. Classical metrics of simple geometric distances (e.g. root mean squared error and Hausdorff distance) $[2,3]$ have been demonstrated not relevant to human visual perception and thus fail to predict the visual difference between a pair of reference and deformed meshes [1].

In order to design an accurate mesh visual distance metric, this paper attempts to reconcile several properties of the human visual system (HVS) with differential geometric quantities. Our contributions are summarized as follows:

- Introduction of an effective approach to the assessment of mesh visual quality (MVQ) based on a novel distance measure between mesh curvature tensors.

- Use of not only curvature values, but also surface principal directions (which have been proven perceptually important) to define the curvature tensor distance. This distance measure seems generic enough to be used in the future in other applications such as mesh segmentation and shape matching.

- Integration of some HVS features in the metric: we introduce a roughnessbased weighting of local visual distance to simulate the visual masking effect, and a processing step similar to Divisive Normalization Transform (DNT) to mimic an important neural mechanism known as adaptive gain control. 
The proposed metric has the potential to be used, for instance, to benchmark a variety of mesh processing algorithms, or to guide the design of new perceptuallyoriented algorithms. The rest of this paper is organized as follows: The relevant research is briefly reviewed in Section 2; Section 3 details the pipeline of the proposed MVQ metric; Section 4 presents some experimental results, including the comparison with state-of-the-art metrics; Finally, we conclude in Section 5.

\section{Related Work and Motivation}

Mesh visual quality assessment. The first perceptually-oriented MVQ metric was introduced by Karni and Gotsman [4] for the evaluation of their mesh compression algorithm. The authors derived a metric by combining errors in both vertex positions and mesh Laplacian coordinates. Corsini et al. [5] developed two perceptual metrics, named respectively $3 D W P M_{1}$ and $3 D W P M_{2}$, for the visual quality assessment of watermarked meshes. The visual distortion is measured as the roughness difference between the original and watermarked meshes. Bian et al. [6] derived a perceptual measure where the visual difference between a pair of meshes is defined as the amount of strain energy required to induce the deformation between them. Lavoué et al. proposed a metric called mesh structural distortion measure $(M S D M)$ [7], which can be considered as an extension of the well-known structure similarity index of $2 \mathrm{D}$ images [8] to the case of 3D triangle meshes. $M S D M$ relates the visual degradation to the alteration of local statistics (i.e. mean, variance and covariance) of mesh curvature amplitudes. An improved multiscale version $M S D M 2$ [9] has been recently proposed, which also integrates a vertex matching preprocessing step to allow the comparison of two meshes with different vertex connectivities.

Motivation. MSDM2 exhibits good correlation with subjective scores [9], though by considering only the modification in mesh curvature amplitudes. We argue that the modification in surface principal directions (as defined by the orthogonal directions of minimum and maximum curvatures) is also important to MVQ assessment, because intuitively these directions imply structural information of the surface and thus should be visually important. Indeed, when drawing a 3D object, one strategy of caricaturists is to draw strokes on these lines of curvatures. In the digital world, surface principal directions have been successfully used for describing [10] and illustrating [11] complex 3D objects, and for guiding a remeshing algorithm [12]. Motivated by the above observation, we introduce a new MVQ metric, named TPDM for Tensor-based Perceptual Distance Measure, that makes use of full information of the mesh curvature tensor, i.e. both curvature amplitudes and principal directions. In the following, we will briefly present a technique for estimating mesh curvature tensors and explain how to obtain curvature amplitudes and principal directions from the tensor.

Curvature tensor estimation. The estimation of mesh curvature tensor is a well-researched problem. So far, the most popular estimation technique is the one from Cohen-Steiner and Morvan [13]. Based on the solid foundation 
of normal cycle theory, they derived an elegant per-vertex curvature tensor estimation. Tensors computed on edges are averaged on a geodesic disk window $B$ of user-defined size to obtain the curvature tensor $\mathcal{T}$ on each vertex $v$ :

$$
\mathcal{T}(v)=\frac{1}{|B|} \sum_{\text {edges } e} \beta(e)|e \cap B| \bar{e} \bar{e}^{t},
$$

where $|B|$ is the area of the geodesic disk, $\beta(e)$ is the signed angle between the normals of the two triangles incident to edge $e,|e \cap B|$ is the length of the part of $e$ inside $B$, and $\bar{e}$ is a unit vector in the direction of $e$ (cf. the inset on right). The minimum and maximum curvature amplitudes $\left(\kappa_{\min }\right.$ and $\left.\kappa_{\max }\right)$ are two

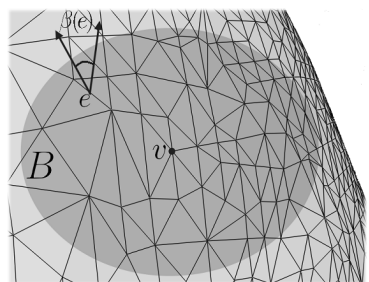
eigenvalues of tensor $\mathcal{T}$, and the principal directions are two eigenvectors $\left(\gamma_{\min }\right.$ and $\left.\gamma_{\max }\right)$. The lines of minimum and maximum curvatures define respectively the directions along which surface normals vary the slowest (e.g. along creases) and the fastest (e.g. across creases), which represent structural features of the surface. In the next section, we will derive a perceptually-oriented distance measure between curvature tensors by incorporating the information from both their eigenvalues and eigenvectors, and use this distance to conduct MVQ assessment.

\section{Curvature Tensor Distance Based MVQ Assessment}

\subsection{Overview of the Pipeline}

Figure 1 illustrates the pipeline of our metric TPDM. First, in order to compare two meshes with potentially different connectivities, we perform a vertex matching step between the two meshes, based on the AABB tree data structure in the CGAL library [14]. The next step is to compute a curvature tensor distance on each local window centered at a vertex. Afterwards, this local tensor distance is weighted by two roughness-based factors, so as to account for the visual masking effect. Finally, we use a surface-weighted Minkowski pooling of the local distances to obtain a global TPDM value.

\subsection{Curvature Tensor Distance}

After the vertex matching step, each vertex $v$ in the reference mesh $\mathcal{M}_{r}$ has a corresponding vertex $v^{\prime}$ on the surface of the distorted mesh $\mathcal{M}_{d}$. The curvature tensors on the two vertices are denoted respectively by $\mathcal{T}$ and $\mathcal{T}^{\prime}$. For the comparison between them, we first establish correspondence relationships between the principal directions and curvature amplitudes of the two tensors. More precisely, for $\gamma_{\min }$ of $\mathcal{T}$, we find the principal direction of $\mathcal{T}^{\prime}$ that has the smallest angular distance to it (this direction is denoted by $\gamma_{1}^{\prime}$ ), and relate $\gamma_{\min }$ to $\gamma_{1}^{\prime}$. Accordingly, $\kappa_{\min }$ of $\mathcal{T}$ is related to the curvature amplitude associated to $\gamma_{1}^{\prime}$ (denoted by $\kappa_{1}^{\prime}$ ). Note that $\kappa_{1}^{\prime}$ and $\gamma_{1}^{\prime}$ can be the maximum curvature and its direction of $\mathcal{T}^{\prime}$. Similarly, the following correspondence relationships are 


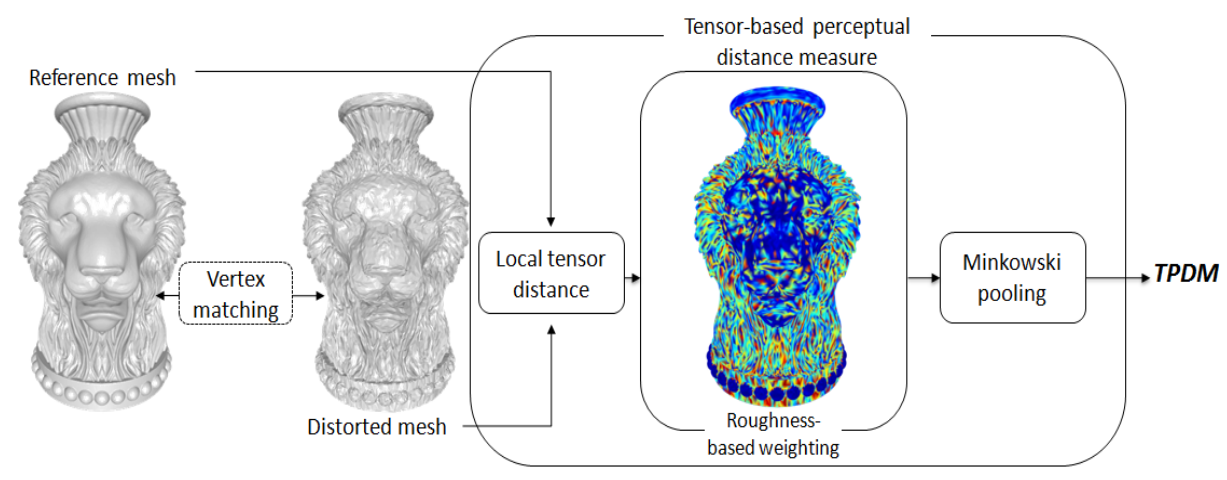

Fig. 1: Block diagram of the pipeline of TPDM (Tensor-based Perceptual Distance Measure). In the roughness map, warmer colors represent larger values.

established: $\kappa_{\max } \rightarrow \kappa_{2}^{\prime}$ and $\gamma_{\max } \rightarrow \gamma_{2}^{\prime}$. We find that the above correspondence based on the minimum angular distance criterion yields better results of MVQ assessment than the straightforward $\min \rightarrow \min / \max \rightarrow \max$ correspondence. In particular, it enhances the stability of TPDM under the situations where the principal directions are severely disturbed after strong deformations and where the values of $\kappa_{\min }$ and $\kappa_{\max }$ are very close to each other.

A local tensor distance $(L T D)$ is computed for each pair of $v$ and $v^{\prime}$ as

$$
L T D=\theta_{\min } \delta_{\kappa_{\min }}+\theta_{\max } \delta_{\kappa_{\max }},
$$

where $\theta_{\min }$ is the angle between $\gamma_{\min }$ and $\gamma_{1}^{\prime}$ (similarly for $\theta_{\max }$ ), and $\delta_{\kappa_{\min }}$ is a Michelson-like contrast of the curvature amplitudes $\kappa_{\min }$ and $\kappa_{1}^{\prime}$, i.e. $\delta_{\kappa_{\text {min }}}=$ $\left|\frac{\kappa_{\min }-\kappa_{1}^{\prime}}{\kappa_{\min }+\kappa_{1}^{\prime}+\varepsilon}\right|$ with $\varepsilon$ a stabilization constant fixed as $5 \%$ of the avarage mean curvature of $\mathcal{M}_{r}$ (similarly for $\delta_{K_{\max }}$ ). Both the differences in curvature amplitudes and in principal directions are involved in the derivation of $L T D$.

\subsection{Roughness-Based Weighting of Local Tensor Distance}

For the development of an effective MVQ metric, we should take into account some HVS features, in particular the visual masking effect. In the context of MVQ assessment, this effect mainly means that a same distortion is less visible in rough regions of the mesh surface than in smooth regions. In order to account for the visual masking effect, our solution is to modulate the values of $L T D_{i, i=1,2, \ldots, N}$ (evaluated at each vertex $v_{i}$ of $\mathcal{M}_{r}$ ) by two roughness-based weights (the rougher the local surface is, the smaller the weights are). The local tensor-based perceptual distance measure $L T P D M_{i}$ is computed as:

$$
L T P D M_{i}=R W_{i}^{(\gamma)} \cdot R W_{i}^{(\kappa)} \cdot L T D_{i},
$$

where $R W_{i}^{(\gamma)}, R W_{i}^{(\kappa)} \in[0.1,1.0]$ are respectively the weights derived from principal directions and curvature amplitudes in 1-ring neighborhood of $v_{i}$. For $R W_{i}^{(\gamma)}$, 
we first project all the principal directions at the 1-ring neighbors on the tangent plane of $v_{i}$, and take the sum of the two angular standard deviations of the projected minimum and maximum curvature directions as the local roughness value. This value is then mapped to $[0.1,1.0]$ to obtain $R W_{i}^{(\gamma)}$. Similarly, to get $R W_{i}^{(\kappa)}$, we compute the ratio of the Laplacian of mean curvature in the 1-ring neighborhood and the mean curvature on $v_{i}$ as the local roughness and map it to $[0.1,1.0]$. It is worth mentioning that the derivation of the roughness weight $R W_{i}^{(\kappa)}$ includes a divisive normalization similar to that in the neural mechanism of HVS that partially explains the visual masking effect [15]. Also note that the vertices in isotropic areas, i.e. where $\kappa_{\min }$ and $\kappa_{\max }$ are close to each other, are treated differently. For these vertices, we set $R W_{i}^{(\gamma)}$ close to 1, and therefore the final weight is dominated by the value of $R W_{i}^{(\kappa)}$. The reason is that in isotropic areas, principal directions are not well-defined and their estimation is not reliable. A roughness map that combines both weights is shown in Fig. 1.

\subsection{Global Perceptual Distance}

The global tensor-based perceptual distance measure TPDM from $\mathcal{M}_{r}$ to $\mathcal{M}_{d}$ is computed as a weighted Minkowski sum of the local distances $L T P D M_{i}$ :

$$
T P D M=\left(\sum_{i=1}^{N} w_{i}\left|L T P D M_{i}\right|^{p}\right)^{\frac{1}{p}},
$$

where $w_{i}=s_{i} / \sum_{i=1}^{N} s_{i}$ with $s_{i}$ one third of the total area of all the incident faces of $v_{i}$, and we set $p=2.5$. The surface-based weighting can, to some extent, enhance the stability of the metric to the variation of vertex sampling density over the mesh surface. Compared to the standard mean-squared error in which $p=2.0$, the choice of $p=2.5$ can increase the importance of the local distances of high amplitude. This is perceptually relevant since the part of mesh with highamplitude distortion has experimentally more impact on the result of subjective assessment. Finally, a cumulative Gaussian psychometric function [16] is applied to bring the TPDM value to the $[0,1]$ interval. More details on the psychometric function will be provided in the next section.

\section{Experimental Results}

In order to verify its efficacy, the proposed metric $T P D M$ has been extensively tested and compared with existing metrics on three subject-rated databases:

- The LIRIS/EPFL general-purpose database ${ }^{1}$ [7]: It contains 4 reference meshes and in total 84 deformed models. The distortion types include noise addition and smoothing, applied either locally or globally on the reference mesh. Subjective evaluations were made by 12 observers.

\footnotetext{
${ }^{1}$ http://liris.cnrs.fr/guillaume.lavoue/data/datasets.html
} 
- The LIRIS masking database ${ }^{2}$ [17]: It contains 4 reference meshes and in total 24 deformed models. The local noise addition distortion included in this database was designed specifically for testing the capability of MVQ metrics in capturing the visual masking effect. 11 observers participated in the subjective tests.

- The IEETA simplification database ${ }^{3}$ [18]: It contains 5 reference meshes and in total 30 simplified models. 65 observers participated in this study.

$T P D M$ has been compared with five state-of-the-art metrics, i.e. the Hausdorff distance $H D[2,3]$, the root mean squared error $R M S[2,3]$, the two roughness-based metrics $3 D W P M_{1}$ and $3 D W P M_{2}$ from Corsini et al. [5], and MSDM2 [9]. The coherence between the distance values produced by the objective metrics and the subjective mean opinion scores $(M O S)$ is measured by using two different kinds of correlation: the Pearson linear correlation coefficient $(P L C C)$ that measures the prediction accuracy of the objective metrics, and the Spearman rank-order correlation coefficient $(S R O C C)$ that measures the prediction monotonicity. Before computing $P L C C$, it is recommended to conduct a psychometric fitting between the objective measures and the $M O S$ values, in order to partially remove the non-linearity between them. In our tests, we apply a cumulative Gaussian psychometric function [16]:

$$
g(a, b, R)=\frac{1}{\sqrt{2 \pi}} \int_{a+b R}^{\infty} e^{-\left(t^{2} / 2\right)} d t,
$$

where $R$ is the raw TPDM value, and the two parameters $a$ and $b$ are obtained through non-linear fitting using the raw TPDM and the corresponding $M O S$ values of the group of Dinosaur models in the general-purpose database. As shown in Fig. 2.(b) and (c), the same psychometric function has been used for the models in the masking and simplification databases.

Tables 1, 2 and 3 present the results on the general-purpose, masking and simplification databases, respectively. In general, TPDM exhibits quite good performance on all the three databases, reflected by its high correlation with subjective scores on most individual models and on the whole repositories. In particular, TPDM has always the highest overall $P L C C$ value (the second last column in the tables), thus the highest prediction accuracy on all the three databases. On the general-purpose database (cf. Table 1), TPDM has the highest $P L C C$ and $S R O C C$ for almost every individual model as well as for the whole repository, and there is much improvement in the overall $P L C C$ compared to $M S D M 2$, the best metric proposed so far. On the masking database (cf. Table 2), although the overall $S R O C C$ of TPDM is not as high as that of $M S D M 2$, we can still conclude that TPDM well captures the visual masking effect, as reflected by the high individual and overall correlation values (all $>80 \%$ ). TPDM has slightly better overall performance than MSDM2 on the simplification database (cf. Table 3). However for the Head model the correlation

\footnotetext{
${ }^{2}$ http://liris.cnrs.fr/guillaume.lavoue/data/datasets.html

${ }^{3}$ http://www.ieeta.pt/ sss/index.php/perceivedquality/repository
} 


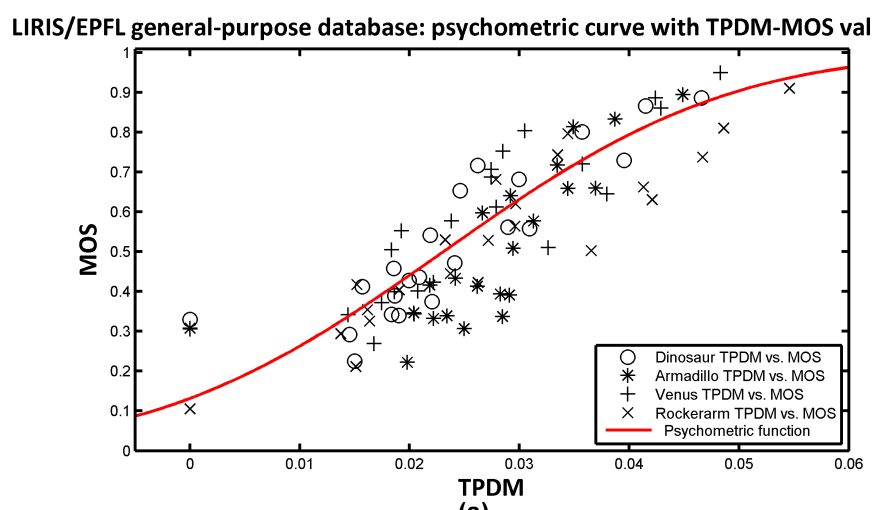

(a)

LIRIS masking database: psychometric curve with TPDM-MOS values

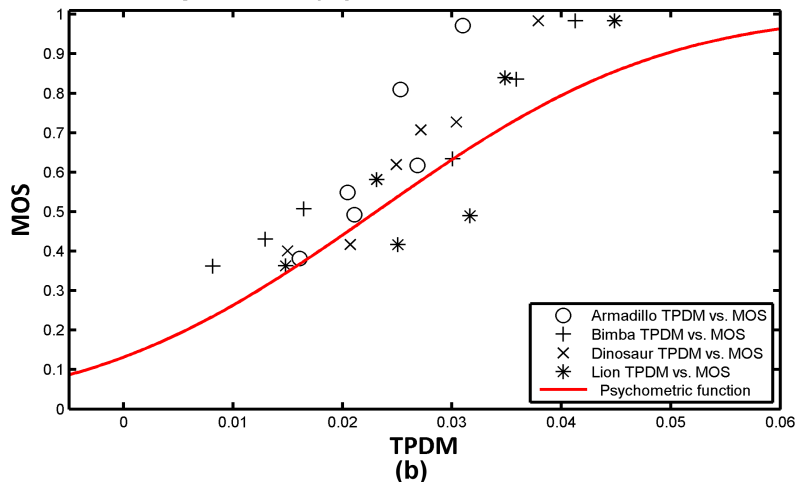

IEETA simplification database: psychometric curve with TPDM-MOS values

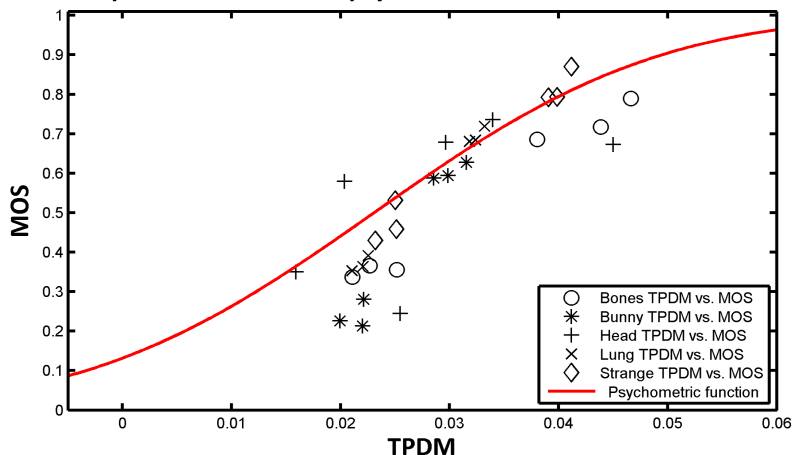

(c)

Fig. 2: Psychometric function curve plotted with TPDM-MOS values of all the reference/distorted models in: (a) the LIRIS/EPFL general-purpose database; (b) the LIRIS masking database; and (c) the IEETA simplification database.

is rather low, and the reason is that TPDM has difficulties in distinguishing the quality of the simplified Heads generated by different mesh simplification algorithms but with the same vertex reduction ratio. 
Table 1: PLCC $\left(r_{p}\right)$ and $S R O C C\left(r_{s}\right)(\%)$ of the different objective metrics on the LIRIS/EPFL general-purpose database.

\begin{tabular}{|c||c|c|c|c|c|c|c|c||c|c|}
\hline \multicolumn{1}{|c||}{ Metric } & \multicolumn{2}{|c|}{ Armadillo } & \multicolumn{3}{c|}{ Dinosaur } & \multicolumn{3}{c||}{ Rockerarm } & \multicolumn{3}{c|}{ Venus } & \multicolumn{3}{c|}{ All models } \\
& $r_{p}$ & $r_{s}$ & $r_{p}$ & $r_{s}$ & $r_{p}$ & $r_{s}$ & $r_{p}$ & $r_{s}$ & $r_{p}$ & $r_{s}$ \\
\hline$H D[2,3]$ & 30.2 & 69.5 & 22.6 & 30.9 & 5.5 & 18.1 & 0.8 & 1.6 & 1.3 & 13.8 \\
\hline$R M S[2,3]$ & 32.2 & 62.7 & 0.0 & 0.3 & 3.0 & 7.3 & 77.3 & $\mathbf{9 0 . 1}$ & 7.9 & 26.8 \\
\hline $3 D W P M_{1}[5]$ & 35.7 & 65.8 & 35.7 & 62.7 & 53.2 & 87.5 & 46.6 & 71.6 & 38.3 & 69.3 \\
\hline $3 D W P M_{2}[5]$ & 43.1 & 74.1 & 19.9 & 52.4 & 29.9 & 37.8 & 16.4 & 34.8 & 24.6 & 49.0 \\
\hline$M S D M 2[9]$ & 72.8 & 81.6 & 73.5 & 85.9 & 76.1 & 89.6 & 76.5 & 89.3 & 66.2 & 80.4 \\
\hline$T P D M$ & $\mathbf{7 9 . 3}$ & $\mathbf{8 5 . 4}$ & $\mathbf{8 9 . 4}$ & $\mathbf{9 2 . 2}$ & $\mathbf{9 1 . 4}$ & $\mathbf{9 0 . 6}$ & $\mathbf{8 7 . 6}$ & 89.9 & $\mathbf{8 4 . 9}$ & $\mathbf{8 5 . 2}$ \\
\hline
\end{tabular}

Table 2: PLCC $\left(r_{p}\right)$ and SROCC $\left(r_{s}\right)(\%)$ of the different objective metrics on the LIRIS masking database.

\begin{tabular}{|c||c|c|c|c|c|c|c|c||c|c|}
\hline \multicolumn{1}{|c||}{ Metric } & \multicolumn{2}{|c|}{ Armadillo } & \multicolumn{2}{c|}{ Bimba } & \multicolumn{2}{c||}{ Dinosaur } & \multicolumn{3}{c||}{ Lion } & \multicolumn{2}{c|}{ All models } \\
& $r_{p}$ & $r_{s}$ & $r_{p}$ & $r_{s}$ & $r_{p}$ & $r_{s}$ & $r_{p}$ & $r_{s}$ & $r_{p}$ & $r_{s}$ \\
\hline$H D[2,3]$ & 37.7 & 48.6 & 7.5 & 25.7 & 31.1 & 48.6 & 25.1 & 71.4 & 4.1 & 26.6 \\
\hline$R M S[2,3]$ & 44.6 & 65.7 & 21.8 & 71.4 & 50.3 & 71.4 & 23.8 & 71.4 & 17.0 & 48.8 \\
\hline $3 D W P M_{1}[5]$ & 41.8 & 58.0 & 8.4 & 20.0 & 45.3 & 66.7 & 9.7 & 20.0 & 10.2 & 29.4 \\
\hline $3 D W P M_{2}[5]$ & 37.9 & 48.6 & 14.4 & 37.1 & 50.1 & 71.4 & 22.0 & 38.3 & 18.2 & 37.4 \\
\hline$M S D M 2[9]$ & 65.8 & $\mathbf{8 8 . 6}$ & 93.7 & $\mathbf{1 0 0}$ & 91.5 & $\mathbf{1 0 0}$ & $\mathbf{8 7 . 5}$ & $\mathbf{9 4 . 3}$ & 76.2 & $\mathbf{8 9 . 6}$ \\
\hline TPDM & $\mathbf{9 1 . 3}$ & $\mathbf{8 8 . 6}$ & $\mathbf{9 7 . 1}$ & $\mathbf{1 0 0}$ & $\mathbf{9 7 . 1}$ & $\mathbf{1 0 0}$ & 86.7 & 82.9 & $\mathbf{8 7 . 1}$ & 87.3 \\
\hline
\end{tabular}

Table 3: PLCC $\left(r_{p}\right)$ and SROCC $\left(r_{s}\right)(\%)$ of the different objective metrics on the IEETA simplification database.

\begin{tabular}{|c||c|c|c|c|c|c|c|c|c|c||c|c|}
\hline \multicolumn{1}{|c||}{ Metric } & \multicolumn{2}{c|}{ Bones } & \multicolumn{2}{c|}{ Bunny } & \multicolumn{2}{c|}{ Head } & \multicolumn{2}{c||}{ Lung } & \multicolumn{3}{c|}{ Strange } & \multicolumn{2}{c|}{ All models } \\
& $r_{p}$ & $r_{s}$ & $r_{p}$ & $r_{s}$ & $r_{p}$ & $r_{s}$ & $r_{p}$ & $r_{s}$ & $r_{p}$ & $r_{s}$ & $r_{p}$ & $r_{s}$ \\
\hline$H D[2,3]$ & 84.8 & $\mathbf{9 4 . 3}$ & 14.3 & 39.5 & 53.0 & $\mathbf{8 8 . 6}$ & 64.9 & 88.6 & 27.4 & 37.1 & 25.5 & 49.4 \\
\hline$M S D M 2[9]$ & 96.7 & 77.1 & 96.3 & $\mathbf{9 4 . 3}$ & $\mathbf{7 9 . 0}$ & $\mathbf{8 8 . 6}$ & 85.3 & 65.7 & 98.1 & $\mathbf{1 0 0}$ & 79.6 & $\mathbf{8 6 . 7}$ \\
\hline$T P D M$ & $\mathbf{9 8 . 9}$ & $\mathbf{9 4 . 3}$ & $\mathbf{9 7 . 9}$ & $\mathbf{9 4 . 3}$ & 63.1 & 65.7 & $\mathbf{9 9 . 9}$ & $\mathbf{1 0 0}$ & $\mathbf{9 8 . 8}$ & 94.3 & $\mathbf{8 6 . 4}$ & $\mathbf{8 6 . 7}$ \\
\hline
\end{tabular}

Figure 3 illustrates the distance maps produced by $T P D M$ and $R M S$ for a noised Bimba model. The map of TPDM is quite consistent with human perception (i.e. the perceived distortion is higher in smooth regions than in rough regions), while the map of $R M S$ is purely geometric. Figure 4 shows an application of our metric in the visual quality assessment of watermarked meshes. The two watermarked models have exactly the same geometric maximum root mean squared error $(M R M S)$ [3] compared to the original mesh, but their visual 

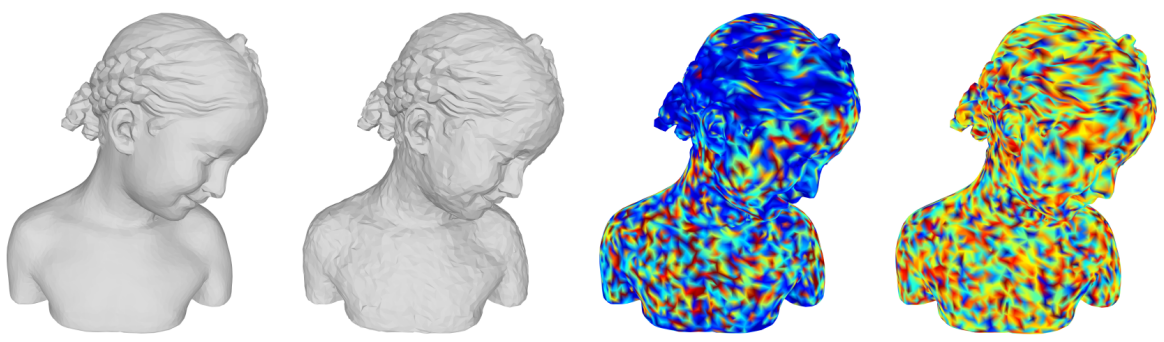

Fig. 3: From left to right: the original Bimba model, the deformed model after uniform noise addition, the distance map of TPDM, and the distance map of $R M S$. In the maps, warmer colors represent higher values.
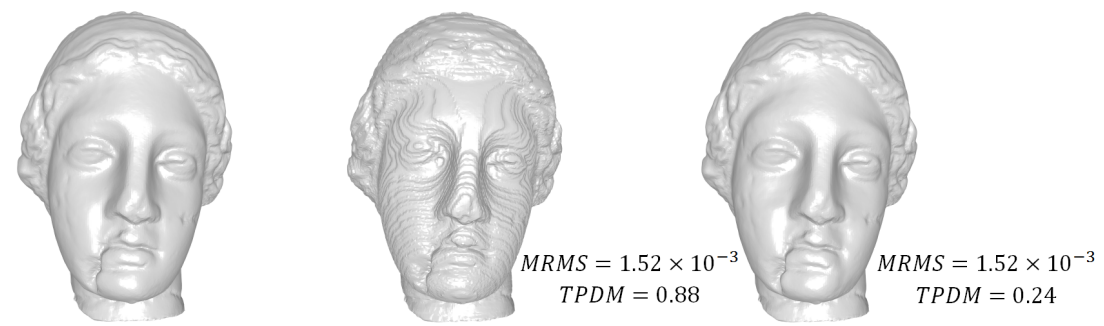

Fig. 4: From left to right: the original Venus model, the model watermarked by the method in [19], and the model watermarked by the method in [20].

quality is quite different. TPDM provides correct MVQ evaluation results that are consistent with a subjective assessment.

\section{Conclusions and Future Work}

A new curvature-tensor-based approach to the objective evaluation of mesh visual quality has been proposed. We show that it is beneficial to use the full information of the curvature tensor for MVQ assessment. The local curvature distance and the local roughness measures that we propose may be found useful in other mesh applications. Experimental results show that our metric TPDM has high correlation with subjective scores and that it slightly outperforms existing metrics. Future work mainly consists of the integration of more HVS features in the metric (e.g. the contrast sensitivity function), the improvement of the roughness measure which at present appears a little noisy, and the development of a curvature-tensor-based visual quality metric for dynamic meshes.

\section{Acknowledgments}

We would like to thank the anonymous reviewers for their helpful and constructive comments. This work has been in part supported by the MOOV3D project of the Minalogic competitive cluster. 


\section{References}

1. Corsini, M., Larabi, M.C., Lavoué, G., Petř́k, O., Váša, L., Wang, K.: Perceptual metrics for static and dynamic triangle meshes. In: Proc. of Eurographics Stateof-the-Art Rep. (2012) 135-157

2. Cignoni, P., Rocchini, C., Scopigno, R.: Metro: measuring error on simplified surfaces. Comput. Graphics Forum 17(2) (1998) 167-174

3. Aspert, N., Santa-Cruz, D., Ebrahimi, T.: MESH: measuring errors between surfaces using the Hausdorff distance. In: Proc. of IEEE Int. Conf. on Multimedia \& Expo. (2002) 705-708

4. Karni, Z., Gotsman, C.: Spectral compression of mesh geometry. In: Proc. of ACM Siggraph. (2000) 279-286

5. Corsini, M., Drelie Gelasca, E., Ebrahimi, T., Barni, M.: Watermarked 3-D mesh quality assessment. IEEE Trans. on Multimedia 9(2) (2007) 247-256

6. Bian, Z., Hu, S.M., Martin, R.R.: Evaluation for small visual difference between conforming meshes on strain field. Journal of Comput. Sci. and Technol. 24(1) (2009) 65-75

7. Lavoué, G., Drelie Gelasca, E., Dupont, F., Baskurt, A., Ebrahimi, T.: Perceptually driven $3 \mathrm{D}$ distance metrics with application to watermarking. In: Proc. of SPIE Electronic Imaging. (2006) 63120L.1-63120L.12

8. Wang, Z., Bovik, A.C., Sheikh, H.R., Simoncelli, E.P.: Image quality assessment: From error visibility to structural similarity. IEEE Trans. on Image Process. 13(4) (2004) 600-612

9. Lavoué, G.: A multiscale metric for 3D mesh visual quality assessment. Comput. Graphics Forum 30(5) (2011) 1427-1437

10. Brady, M., Ponce, J., Yuille, A.L., Asada, H.: Describing surfaces. Comput. Vision, Graphics, and Image Process. 32(1) (1985) 1-28

11. Hertzmann, A., Zorin, D.: Illustrating smooth surfaces. In: Proc. of ACM Siggraph. (2000) 517-526

12. Alliez, P., Cohen-Steiner, D., Devillers, O., Lévy, B., Desbrun, M.: Anisotropic polygonal remeshing. ACM Trans. on Graphics 22(3) (2003) 485-493

13. Cohen-Steiner, D., Morvan, J.M.: Restricted delaunay triangulations and normal cycle. In: Symp. on Computational Geometry. (2003) 312-321

14. Alliez, P., Tayeb, S., Wormser, C.: 3D fast intersection and distance computation (AABB tree). In: CGAL User and Reference Manual. (2012)

15. Li, Q., Wang, Z.: Reduced-reference image quality assessment using divisive normalization-based image representation. IEEE J. Sel. Topics Signal Process. 3(2) (2009) 202-211

16. Engeldrum, P.G.: Psychometric Scaling: A Toolkit for Imaging Systems Development. Imcotek Press (2000)

17. Lavoué, G.: A local roughness measure for 3D meshes and its application to visual masking. ACM Trans. on Appl. Perception 5(4) (2009) 21:1-21:23

18. Silva, S., Santos, B.S., Ferreira, C., Madeira, J.: A perceptual data repository for polygonal meshes. In: Proc. of Int. Conf. in Visualization. (2009) 207-212

19. Cho, J.W., Prost, R., Jung, H.Y.: An oblivious watermarking for 3-D polygonal meshes using distribution of vertex norms. IEEE Trans. on Signal Process. 55(1) (2007) 142-155

20. Wang, K., Lavoué, G., Denis, F., Baskurt, A.: Robust and blind mesh watermarking based on volume moments. Comput. \& Graphics 35(1) (2011) 1-19 